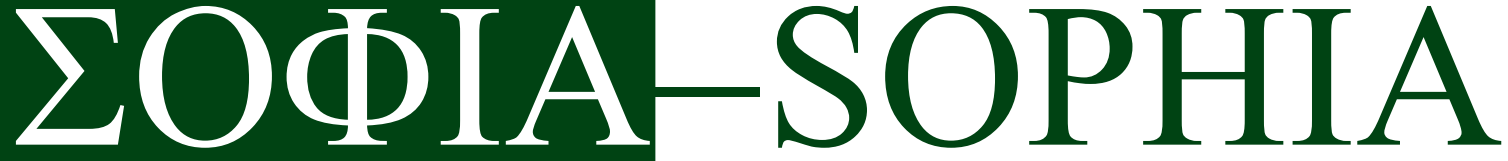

DOI: http://dx.doi.org/10.18634/sophiaj.14v.1i.823

\section{Formación de profesores para una educación matemática en y para la diversidad*}

\section{Teacher training for mathematics education in and for diversity}

\author{
Formação de professores para uma educação matemática \\ e para a diversidade
}

Información del artículo

Recibido: agosto de 2017

Revisado: octubre de 2017

Aceptado: diciembre de 2017

\section{Cómo citar:}

Bermúdez, E.A., Gutiérrez, H., Wagner, G. (2018) Formación de profesores para una educación matemática en y para la diversidad. Sophia, 14(1) 65-74.
Eliécer Aldana Bermúdez** Heiller Gutiérrez Zuluaga*** Graciela Wagner Osorio****

\begin{abstract}
* Investigación realizada con el apoyo de Colciencias, mediante la "Alianza de Instituciones educativas para el Desarrollo de la Educación y Tecnología en Colombia, AIDETC". Proyecto 1: Arquitectura, pedagógica, didáctica y tecnológica para la formación de profesores en y para la diversidad, Grupo Gemauq. Universidad del Quindío, Armenia, Quindío, Colombia.

** Doctor en Educación Matemática, docente de planta de la Universidad del Quindío, integrante del grupo de educación matemática de la Universidad del Quindío “Gemauq”, eliecerab@uniquindio.edu.co, Armenia,Quindío, Colombia

***Magister en Educación, docente de planta de la Universidad del Quindío, integrante del grupo de educación matemática de la Universidad del Quindío "Gemauq", hgutierrez@uniquindio.edu.co,Armenia,Quindío,Colombia.

**** Magister en Educación, docente de contrato de la Universidad del Quindío, integrante del grupo de educación matemática de la Universidad del Quindío “Gemauq”, gwagner@uniquindio.edu.co, Armenia,Quindío,Colombia.
\end{abstract}

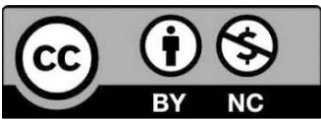

ISSN (electrónico): 2346-0806 ISSN (impreso): 1794-8932 


\title{
Resumen
}

Este artículo tiene como propósito presentar una investigación a partir de un proceso de formación de profesores en educación matemática que atienden poblaciones con deficit cognitivo (Síndrome Down), limitación auditiva o baja visión, y sordera o hipoacúsicos. La formación de profesores se asume teóricamente desde el enfoque ontosemiótico (Godino, Batanero y Font, 2007); las trayectorias de aprendizaje (Clements, y Sarama, 2009 y Simon ,1995); los registros de representación semiótica (Duval, 2004); las situaciones didácticas (Brousseau, 2004), y el conocimiento didáctico del contenido matemático de poblaciones en contextos culturales (Shulman, 1986). La metodología es cualitativa interpretativa, (Medina y Castillo, 2003), porque es un método para observar comportamiento de los profesores y estudiantes, el estudio se realizó con un grupo de 25 profesionales y docentes de apoyo, mediante un proceso de investigación-acción, para lo cual se diseñó un plan, actuación, observación y reflexión de la investigación. Los resultados avizoran de una parte, el grado de motivación generada en los profesionales que atienden estas poblaciones y de otra ofrecerles herramientas para que ellos puedan facilitar el aprendizaje a estos estudiantes y lograr de esta manera una educación matemática en y para la diversidad como puerta de entrada a una educación inclusiva.

Palabras clave: Diversidad, educación matemática, formación de profesores.

\begin{abstract}
This communication aims to present a research based on a process of teacher training in mathematics education that addresses populations with cognitive impairment (Down Syndrome), auditory limitation or low vision, and deaf or hard of hearing. Teacher training is theoretically assumed from the ontosemiotic approach (Godino, Batanero and Font, 2007); The Learning Paths (Clements and Sarama, 2009 and Simon, 1995); The registers of semiotic representation (Duval, 2004); The didactic situations (Brousseau, 2004), and the didactic knowledge of the mathematical content of populations in cultural contexts (Shulman, 1986). The methodology is qualitative interpretive, (Medina and Castillo, 2003), because it is a method to observe behavior of teachers and students, the study was conducted with a group of 25 professionals and support teachers, through a process of action research, For which a plan, action, observation and reflection of the research was designed. The results provide an insight into the degree of motivation generated in the professionals who care for these populations, and on the other hand, offer them tools so that they can facilitate the learning of these students and thus achieve a mathematical education in and for diversity as a gateway entry to inclusive education.
\end{abstract}

Keywords: Teacher training, mathematics education, diversity.

\section{Resumo}

O objetivo deste artigo é apresentar uma pesquisa baseada em um processo de formação de professores em educação matemática que sirva populações com déficit cognitivo (Síndrome de Down), limitação auditiva ou baixa visão e surdez ou perda auditiva. O treinamento do professor é assumido teoricamente a partir da abordagem ontosemiótica (Godino, Batanero e Font, 2007); as trajetórias de aprendizagem (Clements e Sarama, 2009 e Simon, 1995); os registros da representação semiótica (Duval, 2004); as situações didáticas (Brousseau, 2004) e o conhecimento didático do conteúdo matemático das populações em contextos culturais (Shulman, 1986). A metodologia é interpretativa qualitativa, (Medina e Castillo, 2003), porque é um método para observar o comportamento dos professores e alunos, o estudo foi realizado com um grupo de 25 profissionais e professores de apoio, através de um processo de pesquisa-ação, para o qual foi planejado um plano, ação, observação e reflexão da pesquisa. Os resultados mostram, por um lado, o grau de motivação gerado pelos profissionais que servem essas populações e, por outro lado, oferecem-lhes ferramentas para que possam facilitar o aprendizado para esses alunos e, assim, conseguir uma educação matemática na diversidade e como uma porta entrada para uma educação inclusiva.

Palavras-chave: Diversidade, educação matemática, formação de professores. 


\section{Introducción}

Esta investigación tiene como propósito desarrollar un proceso de formación con profesionales y docentes de apoyo que no necesariamente son licenciados en matemáticas y que por tanto requirieren de una actualización y perfeccionamiento en didáctica de las matemáticas. El propósito es que adquieran algunos instrumentos del conocimiento para que puedan atender poblaciones en condición de educación inclusiva (Ministerio Educación Nacional, Men, 2017); centrado en el caso particular de personas que presentan capacidades diferenciadas como: Deficit cognitivo (Síndrome Down), limitación auditiva y/o baja visión, y sordos y/o hipoacúsicos para elaborar un ambiente didáctico que privilegie las condiciones de estas poblaciones como potencial de aprendizaje de las matemáticas y los recursos tecnológicos como instrumentos necesarios para ofrecer multiplicidad de formas de exploración con las matemáticas a esta poblaciones sobre la aprehensión de los fenómenos numéricos, geométricos, métricos, variacionales y aleatorios.

En este sentido el proceso docente educativo construye un entorno propicio: "El cual debe ser el que se ajuste y adapte a las particularidades de todos y todas, identificando y trabajando en las barreras que existen para el aprendizaje y la participación (Men, 2017, p. 5)". Se trata de realizar un proceso de formación y actualización docente ligado a los aspectos socioculturales de la educación matemática para ofrecer a los niños, niñas, jóvenes y adolescentes una educación de mejor calidad y de igualdad de oportunidades, mediante políticas institucionales de cara al siglo XXI.

En las últimas décadas, producto del análisis y reflexión sobre la importancia del rol del docente en los procesos de transformación de las concepciones, las prácticas, las actitudes que inciden en forma directa en la educación, se han generado reformas educativas en varios países de América Latina y demuestran que esto no ha sido suficiente y es necesario cambiar la formación tradicional de los docentes, centrada en lo disciplinar, en especial cuando se requiere formar docentes de matemáticas que reconozcan la diversidad de los contextos, avances tecnológicos, los ritmos de aprendizaje de los estudiantes, las situaciones económicas, sociales, políticas de los estudiantes y de las comunidades donde se desarrolla el sistema educativo, para que de esta forma el docente actúe a partir de dicho reconocimiento y facilite el acceso al conocimiento matemático.

Una de las tensiones se presenta entre los sistemas educativos y la política educativa a nivel mundial, regional y local, es lograr que se promueva la implementación de diferentes estrategias y la creación de ambientes educativos. Entonces ¿En qué forma las propuestas de actualización y formación de docentes de matemáticas en ejercicio, incorpora estas particularidades, las hace visibles y trabaja con ellas? ¿Con qué tipo de currículo se encuentra el estudiante para profesor de matemáticas, para que puedan responder a uno de los mayores desafíos que tienen los docentes en el siglo XXI, hacer posible que el derecho a la educación, se convierta en una realidad para todos, es decir, que a pesar de la diversidad de géneros, culturas, ritmos de aprendizaje, condiciones económicas, historias de vida, discapacidades, lenguajes y muchos otros se puedan generar las condiciones sociales, afectivas y académicas para aprender matemáticas?

El reto es para todo sistema educativo que se proponga construir en los estudiantes para profesor de matemáticas y promover en los docentes en ejercicio, con el propósito de superar la necesidad social y cultural de transformar las prácticas educativas, por las que se accede al conocimiento matemático, que originan que el área de matemáticas sea considerada la que mayores complicaciones crea para estudiante y docentes, pues es la que tiene los más altos índices de fracaso escolar produce en estudiantes y personas en general, rechazo, desánimo y falta de motivación para su aprendizaje.

Ante lo planteado, la propuesta de acción es la realización de la investigación enmarcada en la formación de docentes de matemáticas para el desempeño en la educación intercultural. Por tanto, la presente investigación propone hacer visible las necesidades, posibilidades y potencialidades de las poblaciones que se encuentran en condición de diversidad sensorial y cognitiva, a partir del siguiente interrogante ¿Qué aspectos didácticos deben ser tenidos en cuenta para el proceso de formación didáctica a profesores que atienden poblaciones en condición de discapacidad intelectual (síndrome Down), limitación auditiva (hipoacúsicos, sordos) y limitación visual (baja visión y ciegos), de instituciones de Educación formal y no formal en ArmeniaColombia?

\section{Marco teórico}

En primer lugar, la educación inclusiva en el contexto de este artículo es asumida de acuerdo con el (MEN, 2017:5) como:

Un proceso permanente que reconoce, valora y responde de manera pertinente a la diversidad de características, necesidades, intereses, posibilidades y expectativas de todos los niños, niñas, adolescentes, jóvenes y adultos, con pares de su misma edad, a través de prácticas, políticas y culturas que eliminan las barreras para el aprendizaje y la participación; garantizando en el marco de los derechos humanos cambios y modificaciones en el contenido, los enfoques, las estructuras y las estrategias. (MEN, 2017, p. 4 - 5), 
En el documento del MEN: "Enfoque de educación inclusiva en la actualización pedagógica de los Educadores" (2017), se explican seis principios básicos de la educación inclusiva. De acuerdo con la experiencia vivida en el programa de formación con los docentes participantes, se hizo una adaptación de la figura, donde se incorporan otros elementos relevantes que entran en juego para enseñar a las personas con limitaciones sensoriales y/o cognitivas, para facilitar el aprendizaje a estas poblaciones y lograr una verdadera inclusión. Ver gráfica 1

Gráfica 1: Principios básicos de la Educación Inclusiva

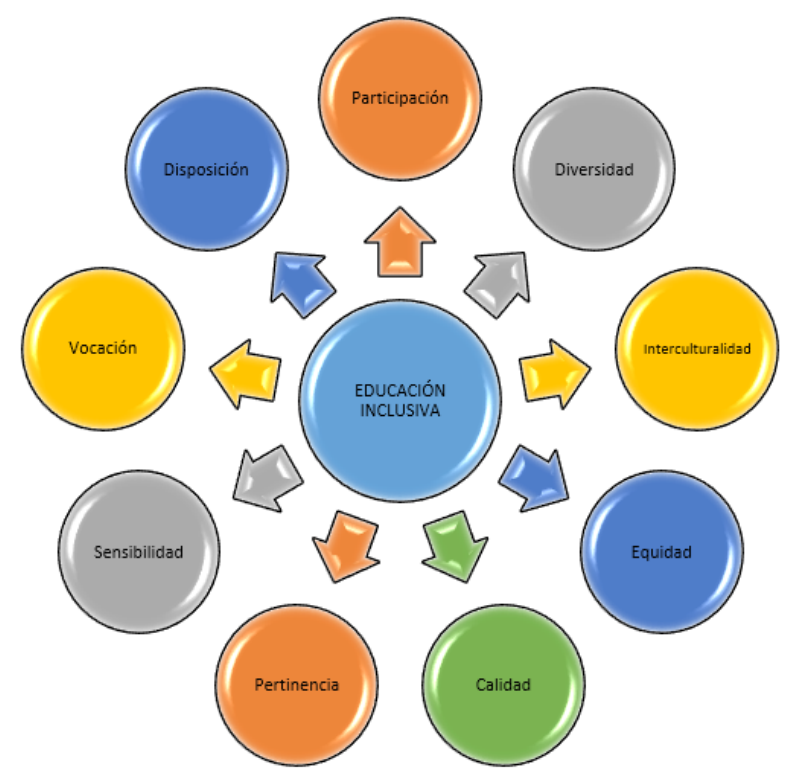

Fuente: Adaptación de “principios en sus apartados en su mayoría fueron tomados de manera literal de los Lineamientos Política de educación superior inclusiva, MEN (2017).

En relación con la formación de profesores se considera como el conjunto de referentes curriculares en contextos de diversidad, desde el enfoque ontosemiótico (Godino, Batanero, y Font, 2007); las Trayectorias de Aprendizaje (Clements, y Sarama, 2009 y Simon ,1995); los registros de representación semiótica (Duval, 2004); las situaciones didácticas (Brousseau, 2004), en los planteamientos de (Shulman, 1986), proponen que el conocimiento para realizar la práctica de enseñar matemáticas debe incorporar el conocimiento del entorno cultural del aula y de las condiciones de sus poblaciones en su contexto. Al respecto, (Bruno y Noda. 2010:146-147) dicen que: "Los profesores que atienden estudiantes especiales tienen una fuerte formación en aspectos psicológicos y pedagógicos, pero no han recibido formación en contenidos didácticos de áreas curriculares, lo que les lleva a tener inseguridades en el tratamiento de los diferentes contenidos".

El estudio en lo social cognitivo (Vigotsky, 1979), plantea que, de hecho, el funcionamiento individual está determinado exclusivamente por el funcionamiento social y que la estructura de los procesos mentales de un individuo refleja el medio social del cual se deriva; en las manifestaciones de competencia cognitiva y cultural de las personas con limitaciones cognitivas, (López Melero, 1999). En particular, (uiz, 2013) afirma que el escolar con deficit cognitivo (SD), no basta con que asista a la escuela, sino que se le debe dar oportunidades para que aprovechen al máximo el espacio escolar y que esto se logra cuando cuentan con la atención necesaria, una actitud favorable y una adecuada formación de los educadores; la comunidad sorda se fundamenta en que la conexión con el mundo a través de la visión y el uso de una lengua de signos confiere rasgos de identidad propios, y en las personas con baja visión la educación ha de ponerse a tono y servirse de las ventajas que los sistemas cibernéticos y la inteligencia artificial le pueden proporcionar, (Blázquez/Lucero, 2002).

\section{Materiales y métodos}

La metodología en la cual se fundamenta esta investigación es de tipo cualitativo interpretativo (Medina \& Castillo, 2003) porque utiliza un método apropiado para mirar los matices de comportamiento de los profesores y estudiantes, genera afirmaciones e interrogantes reflexivos con base en las evidencias a partir del análisis y de los objetivos de la investigación. Mediante las comunidades de práctica se pretende obtener secuencias didácticas mediadas por la incorporación tecnológica, para el desarrollo matemático inicial de las poblaciones objeto del estudio.

A partir del análisis y de los resultados obtenidos de la investigación, se espera validar el marco de referencia teórico y metodológico, del trabajo en red y pensando en la formación de profesores, y unos materiales tecnológicos, pedagógicos y 
didácticos, que ofrezcan al profesor las herramientas necesarias para enseñar a las personas con limitaciones sensoriales y/o cognitivas y por tanto facilitar el aprendizaje a estas poblaciones, de acuerdo con las necesidades y expectativas, y de esta manera lograr una verdadera inclusión en el sistema educativo convencional.

El enfoque ha sido de una metodología de investigación - acción orientada por las fases: 1. Identificación de las poblaciones objeto de la investigación y de ambientes naturales de enseñanza. 2. Elaboración y aplicación de un diagnóstico, para conocer las necesidades y expectativas de los profesores. 3. Desarrollo de laboratorios didácticos para la aproximación epistemológica a las tres frentes de interés, sobre los objetos matemáticos de estudio, el marco teórico y metodológico de la investigación. 4. Diseño de situaciones didácticas, que relaciona los pensamientos numéricos, espacial - métrico, variacional y aleatorio, en concreto, la comprensión/construcción de los conceptos matemáticos básicos en las poblaciones con deficit cognitivo, sordera, y ceguera. 5. Actualización docente mediante prácticas durante el seminario sobre secuencias didácticas en las tres poblaciones, tomando como eje articulador objetos reales para generar motivación en los profesores por las matemáticas y su enseñanza. 6 . Aplicación de la teoría y la práctica de profesores con los sus estudiantes en ambientes naturales de enseñanza y de aprendizaje. 7. Validación de las secuencias didácticas elaboradas por los mismos profesionales y docentes de apoyo, para socializar los resultados obtenidos ante la comunidad académica, mediante la presentación de un producto como resultado de la actualización y perfeccionamiento docente.

\section{Resultados y discusión}

Con el propósito de configurar el objetivo general de esta investigación en la formación de profesores que atienden poblaciones con capacidades diferenciadas a nivel de: déficit cognitivo (SD), limitación auditiva (hipoacúsicos, sordos) y limitación visual (baja visión y ciegos) de instituciones educativas, se realizó el análisis de un instrumento diagnóstico de veinte preguntas de tipo cualitativo y cuantitativo, de respuestas abiertas que brindan la apreciación personal sobre fortalezas y debilidades que tienen los docentes de apoyo en el campo de la enseñanza de las matemáticas a estudiantes en condiciones de diversidad.

En relación con la formación profesional, de acuerdo con esta variable se observa que la gran mayoría de los docentes no manejan adecuadamente el área matemática en cuanto a procesos de conceptualización, por tal motivo se pueden generar falencias en la enseñanza de los conceptos básicos en las diversas poblaciones de diversidad, asimismo los educandos pueden presentar inseguridad al enfrentar el contexto matemático o desarrollo propio del aprendizaje.

De otra parte, reconocen no tener una buena experiencia durante su proceso de formación en cuanto a la forma de enseñanza y de aprendizaje de las matemáticas que ellos mismos tuvieron que aprender y consideran, porque no tiene el saber disciplinar para desarrollar pensamiento matemático en estas tres poblaciones a las cuales se hace referencia a lo largo de este documento. Reconocen la formación y actualización en aspectos generales de la formación de un profesional de apoyo y docente de apoyo, pero desconocen elementos de actualización en cuanto al saber propio de las disciplinas y en particular de las matemáticas, los resultados permitieron identificar condiciones y necesidades de los profesores para la enseñanza en contextos de diversidad y reconocer los recursos (cognitivos, físicos, comunicativos, emotivos, sociales) desde las dificultades de comunicación, de representación y de resolución de problemas en el momento de enseñar conceptos matemáticos en el aula.

A partir del diagnóstico realizado en las fases consecutivas del proceso se diseñaron diferentes secuencias didácticas desde enfoques teóricos diferentes. A continuación, se muestran algunos ejemplos:

\section{Secuencia didáctica 1: conteo}

Objetivo: Mediante el Geoplano (gráfica 2) El estudiante podrá explorar los objetos geométricos para reconocer la forma, el tamaño y realizar procesos de asociación; correspondencia y conteo de objetos.

Gráfica 2. Juego de material concreto MathGalileo

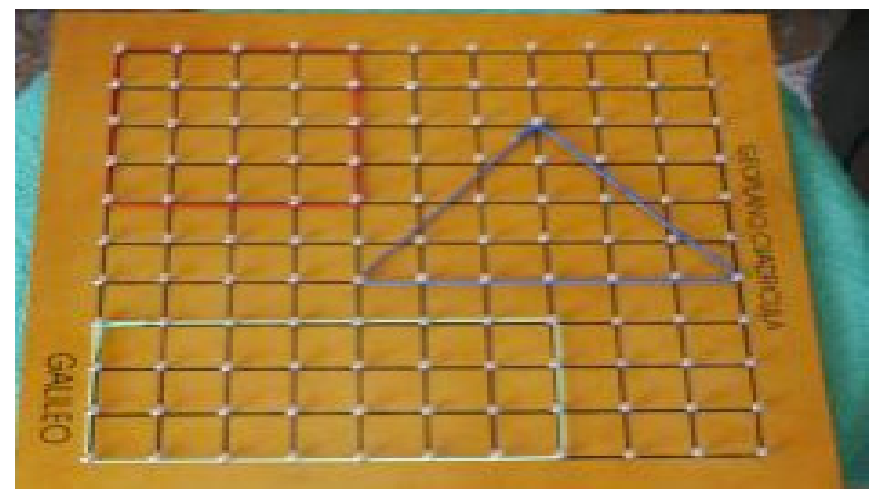

Fuente: los autores 
Variable didáctica. Conteo (Relación uno a uno, uno a muchos, muchos a uno)

\section{Gestión de la clase}

Se hace entrega de un geoplano. Este material permite conformar un lugar geométrico desde una unidad mínima hasta la agrupación de la misma, para lo cual necesitan adicionar, lana, piola o bandas elásticas para realizar la asociación puntilla, unidad, agrupación de unidades y forma. Lo anterior permite solicitar a los participantes que realicen la actividad.

\section{Procedimiento}

1. Se presenta al niño el tablero del Geoplano para que realice un proceso de sensibilización con el material de apoyo (Tocar - Sentir - Observación )

2. Construya figuras (formas) diferentes utilizando el Geoplano y las bandas elásticas, direccionado por el facilitador (ver gráfica 3).

Gráfica 3. Figuras geométricas construidas con las bandas
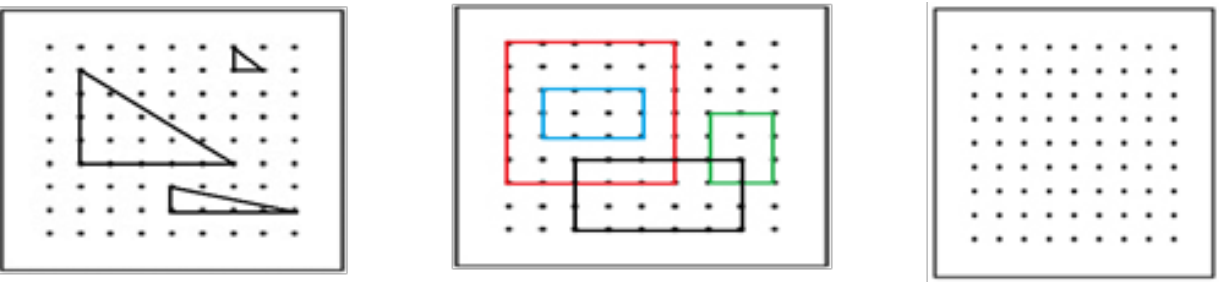

Fuente: los autores

3. El facilitador realiza preguntas de asociación contextual al estudiante, como ¿Qué dibujo? ¿Qué significa? Con el fin de determinar el concepto de figura cerrada o figura abierta.

4. Ahora se les solicita a los estudiantes que realicen con el Geoplano y las bandas elásticas lo siguiente (ver gráfica 4).

Gráfica 4. Otras figuras geométricas construidas
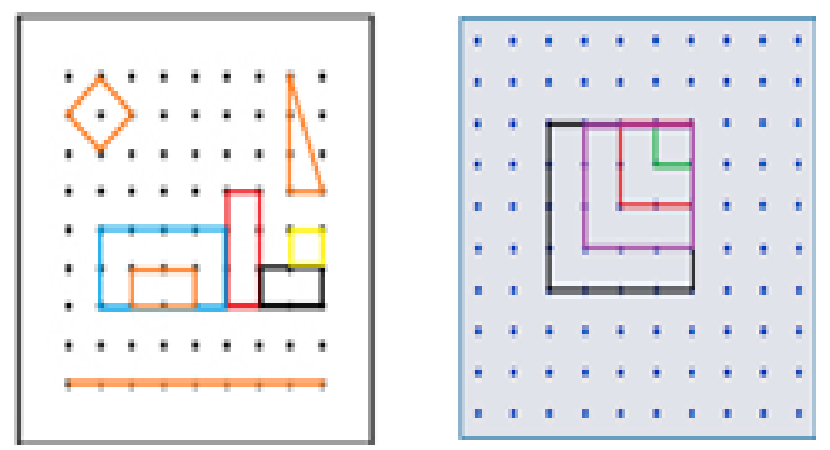

Fuente: los autores

Lo anterior nos permite realizar preguntas de la siguiente manera: ¿Cómo se llama la figura plana que dibujaron? ¿Son del mismo tamaño? ¿Son iguales? ¿Cuál es más grande? Esta descripción espacial del objeto nos permite preguntar ¿Cuántos puntos hay encerrados? Respectivamente, ¿Cuántos puntos se cubrieron con la banda elástica? Como reto direccional se le puede solicitar al estudiante que dibuje nuevamente las figuras, pero de tal manera que (ver ilustración) para realizar preguntas de cuál es el color del "cuadrado" más pequeño; más grande. El cuadrado rojo está adentro o afuera, se puede llenar la figura de color negro con figuras de color verde (Correspondencias - Inclusión). 


\section{Análisis de la secuencia}

Los participantes logran sin dificultad trasladar las figuras de la fotocopia al Geoplano y en el momento en el que el facilitador hace preguntas como cuántas puntillas hay en el interior, ellos responden de manera acertada, dándonos a entender que reconocen características como interior de una figura o exterior de una figura. Además, construyen algunas figuras considerando elementos como número lados, la región interior y exterior de la construcción realizada.

\section{Secuencia didáctica 2: identificación de las formas en el entorno}

Objetivo: Reconocer formas, tamaño, colores, propiedades, relaciones y representaciones de algunos conceptos geométricos, a partir de la observación y la manipulación de objetos del entorno cercano al estudiante.

Recursos: Entorno del estudiante, papel, y colores, dominó de figuras geométricas.

\section{Variable didáctica: formas, tamaños colores de algunas figuras geométricas}

\section{Gestión de la clase:}

El espacio del niño está rodeado de elementos geométricos, con significados concretos: puertas, ventanas, pisos, tableros, pupitres. En su entorno cotidiano, en casa, su ciudad, colegio y parques, aprende a organizar mentalmente y a orientarse en el espacio. Este es el contexto apropiado para desarrollar las enseñanzas geométricas, de manera significativa.

A partir de estas situaciones y mediante manipulación, y visualización de objetos se puede fomentar el desarrollo de los conceptos geométricos.

\section{Procedimiento}

- El profesor busca que los estudiantes encuentren las diferentes formas en el entorno y establezcan una relación entre el de la geometría y el medio real, donde cobran sentido estos elementos geométricos.

- A partir de relatos, orienta a los estudiantes para que observen esos elementos en medio físico real.

- A través de preguntas se pretende que los estudiantes establezcan una relación entre la geometría y el medio real, donde cobran sentido estos elementos geométricos.

- Mediante la instrucción anterior, les plantea a los estudiantes que hagan representaciones de los diferentes objetos geométricos que ellos consideran presentes en el medio observado.

- Para afianzar las representaciones de algunas figuras geométricas se les plantea el juego de dominó por parejas. Este juego se trabaja con todas las poblaciones, haciendo modificaciones para cada una de ellas. Para el caso de la población ciega o con baja visión, cada figura de cada ficha del dominó, con una rodaja, se repuja su contorno, para que el estudiante ciego pueda identificar cada ficha. (ver gráfica 5)

Gráfica 5. Fichas de dominó

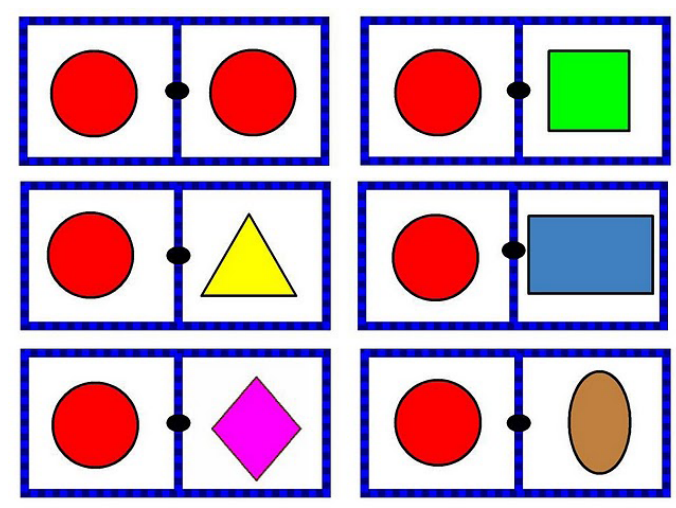

Fuente: Recuperado de: http://miescueladivertida.blogspot.com.co/2011/09/juego-de-domino-figuras-geometricas.html 


\section{Análisis de la secuencia}

Los participantes logran sin dificultad identificar las figuras del dominó, asociando cada una con su respectivo nombre y reconocen las semejanzas y diferencias entre ellas. Por último, realizan el juego por parejas.(ver gráfica 6).

\section{Secuencia didáctica 3: polígonos y poliedros con material didáctico (politroquelados)}

Gráfica 6. Modelo de polígono

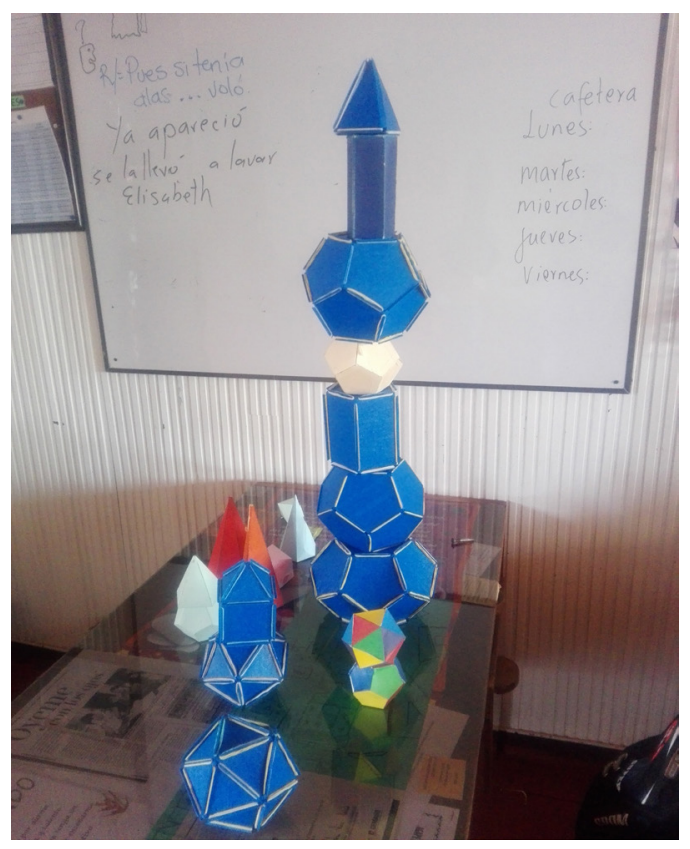

Fuente: los autores

Objetivo: Identificar las características de diferentes poliedros con en su forma plana y espacial.

\section{Variable didáctica: polígonos y poliedros}

\section{Gestión de la clase}

A través de esta actividad se busca que el estudiante reconozca el número de caras y las clases de polígonos que forman un poliedro. Además, se elaboran poliedros con materiales didácticos y se determinan los nombres y las fórmulas matemáticas para hallar áreas laterales, totales y volumen de los poliedros regulares.

\section{Procedimiento}

1. Se presentan las clases de polígonos en materiales didácticos.

2. Se escriben en el tablero los nombres de los poliedros que se pueden armar con los polígonos.

3. Se entrega por grupos paquetes de polígonos diferentes figuras: cuadrados, rectángulos, triángulos equiláteros e isósceles, pentágonos, hexágonos.

4. Armar prismas y pirámides de diferentes bases, reconociendo características y propiedades de cada uno.

5. Armar los poliedros regulares: tetraedros, octaedros, dodecaedros, e icosaedros. Reconocer las características de cada uno.

\section{Análisis de la secuencia}

Se pudo evidenciar en los estudiantes el poliedro que más dificultad les causo construir. A través de un test en tres columnas los estudiantes relacionan el nombre del poliedro con su forma plana y espacial. Además, los estudiantes plantearon diferentes procedimientos para hallar el área lateral, total y volumen de prismas, pirámides, y los poliedros regulares. 


\section{Secuencia didáctica 4: contenidos digitales móviles}

Objetivo: Reconocer diferentes aplicaciones móviles como herramienta de apoyo en matemáticas.

\section{Variable didáctica: entornos digitales}

\section{Gestión de la clase}

A través de esta actividad se busca que el estudiante reconozca aplicaciones móviles o app (en inglés) diseñadas para ser ejecutadas en teléfonos inteligentes, tabletas y otros dispositivos móviles, las cuales permiten una tarea concreta, a nivel profesional, educativo y de acceso a servicios.

\section{Procedimiento}

Actividades desarrolladas:

1. Definición de aplicación móvil.

2. Distribución: Android (Google Play), Windows y Apple.

3. Trabajo en equipos: se organizan en grupos de 3 personas, se les entrega a cada grupo una tablet con el siguiente entorno para trabajar una guía para el reconocimiento de herramientas digitales y algunas aplicaciones para educación en Android.(ver gráfica 7)

Gráfica 7. Aplicaciones móviles para la construcción de figuras geométricas y desarrollo de operaciones matemáticas.

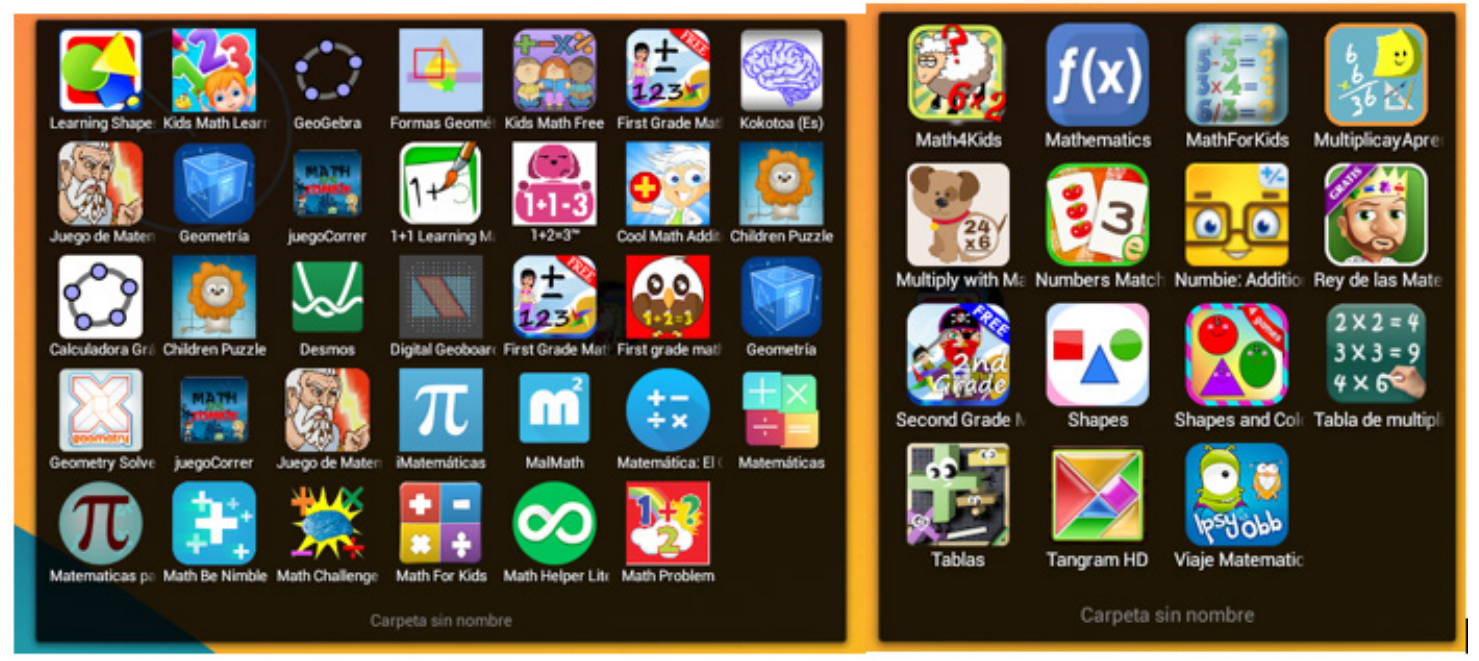

Fuente: los autores

\section{Análisis de la secuencia}

Se pudo evidenciar en los estudiantes que el uso de los entornos digitales como herramienta de apoyo, favorece el aprendizaje de las matemáticas iniciales y es un elemento determinante para reconocer propiedades de conceptos matemáticos.

Las secuencias diseñadas y trabajadas contribuyen al desarrollo del pensamiento matemático, en algunas nociones matemáticas que hacen parte de los tipos de pensamiento numérico, espacial, métrico, aleatorio y variacional, que se desarrollaron con los docentes participantes del programa de formación de tal forma que pudieran ser llevada al aula de clase, donde los docentes tuvieran la oportunidad de usar tanto material manipulable, como entornos digitales.

\section{Conclusiones}

Las problemas que tienen los profesionales de apoyo con estas poblaciones son: ausencia de representación de los conceptos matemáticos, dificultad para establecer relaciones entre formas de representación de un concepto, poca aplicabilidad de los conceptos matemáticos, falta de habilidad para plantear estrategias de resolución de problemas, carencias en el manejo del braille, ausencia de material especializado y conflicto para resolver problemas que involucran la lógica, dificultades en lengua de 
señas, barreras de comunicación que dificultan la interacción con sus pares oyentes, falta de comprensión, y de aplicación de los conceptos, ausencia de significados de los conceptos matemáticos.

La mayoría de docentes de apoyo que participaron en el programa de formación en educación matemática, que atienden poblaciones con déficit cognitivo (Síndrome de Down), limitación auditiva o baja visión, y sordera o hipoacústicos, no son profesionales en el área de matemáticas, sin embargo, mostraron gran interés en la apropiación de los diferentes marcos teóricos y metodológicos trabajados, para incorporarlos en sus aulas de clase y propiciar espacios que conlleven al cumplimiento de los objetivos propuestos de la educación inclusiva.

El encuadre epistemológico realizado con los docentes les permitió reconocer algunas teorías para poder mediar en parte las dificultades que tenían de manifiesto, reconocer la didáctica de las matemáticas como disciplina científica y no con una visión únicamente instrumentalista. El grado de motivación que alcanzaron los profesionales y docentes de apoyo superaron las expectativas previstas y fue demostrado por la socialización de sus reflexiones y trabajos de campo con otros docentes y estudiantes desde sus propios escenarios naturales de enseñanza y de aprendizaje de las matemáticas.

El recorrido por la didáctica desde los diferentes pensamientos, el uso e implementación de recursos como medio para la transposición didáctica para la enseñanza de las matemáticas generó en los docentes participantes mayor interés por las matemáticas, así como una postura diferente en el momento de transferir a los estudiantes las nociones matemáticas abordadas en las diferentes secuencias didácticas.

\section{Referencias bibliográficas}

Blázquez, L. (2002). Los medios y recursos en el proceso didáctico. Madrid: Prentice Hall.

Brousseau, G. (2004). Research in mathematical education, Regular Lecture en el 10th. International Congress on Mathematics Education Dinamarca: ICME10

Bruno, A y Noda, A. (2010). Necesidades educativas especiales en matemáticas. El caso de personas con síndrome de Down. En Moreno, Estrada, Carrillo y Sierra. (eds) Investigación en Educación Matemática pp. 141-162. Lleida: SEIEM.

Clements, D y Sarama, J. (2009). Learning and teaching early math: The learning trajectories approach. New York: Routledge.

Duval, R. (2004). Semiosis y Pensamiento Humano Registros Semióticos y Apendizajes Intelectuales. Santiago de Cali: PeterLang.

Godino, J., Batanero, C y Font, V. (2007). Un Enfoque Ontosemiótico de la Instrucción y la Cognición Matemática. ZDM. The International Journal on Mathematics Education, 39(1), 127-135.

López, M. (1999). Aprendiendo a conocer a las personas con síndrome de Down. Málaga: Aljibe.

Medina, A y Castillo, S. (2003). Metodología para la realización de proyectos de investigación y tesis doctorales. Mathematics Education, 39, 127-135.

MEN. (2017). Enfoque de educación inclusiva en la actualización pedagógica de los educadores. Bogotá: Ministerio de Educación Nacional.

Ruiz, E. (2013). Cómo mejorar la atención de los niños con síndrome de down. I Revista de Sindrome de Down, 30, 63-75

Shulman, L.S. (1986). Paradigms and research programs for the study of teaching. En M.C Wittrock (Ed) Handbook of research on teaching. (Pp. 3-36) New York: Macmillan.

Simon, M. (1995). Reconstructing mathematics pedagogy from a constructivist perspective. Journal for Research in Mathematics Education, 26 (2), 114-145.

Vigotsky, L. (1979). El desarrollo de los procesos psicológicos superiores. Barcelona: Crítica. 